\title{
II. COMMISSION DES COUCHES EXTERIEURES DU SOLEIL
}

Président: M. Y. Öhman.

Membres: MM. Abetti, Allen, Blaha, Mme Brück, MM. Bruzek, Coutrez, Das, Mme d'Azambuja, M. d'Azambuja, Mlle Dodson, MM. Dollfus, dos Reis, Ellison, J. W. Evans, Gnevyshev, Gullón, Kiepenheuer, Kleczek, Krat, Link, McMath, Macris, Miyamoto, Mohler, R. Müller, Mustel, Narayan, Newton, Nicholson, Notuki, Pettit, Pikelner, Rahman, Richardson, Roberts, Romañá, Saha†, Sellers, Severny, Shklovsky, Suemoto, Svestka, Thomsen, Unsöld, van de Hulst, Vyazanitsyn, Waldmeier.

\section{it a. Sous-commission pour la Cinématographie des Phénomènes}

\section{CHROMOSPHÉRIQUES}

Président: M. W. O. Roberts.

Membres: MM. d'Azambuja, Gnevyshev, McMath, Severny, Waldmeier.

\section{GeNERAL}

Since the last General Assembly the solar activity has passed a deep minimum. This has not meant a reduced activity of the solar physicists, however, according to the many valuable reports received for the Draft Report. In fact, during the last years considerable work has been done in discussing previous observations and in improving theory and methods of observation. One example is the extremely important volume The Sun, edited by G. P. Kuiper. Particularly valuable for the work of the commission are Chapters 5,6 and 9 of this volume.

Since the last General Assembly, many solar observatories have improved their instrumental equipment and made preparations for an intensified solar patrol during the new sunspot cycle. Considerable interest has been created also by the special plans for the Geophysical year I957-58.

\section{IMPROVEMENTS IN EQUIPMENT}

The number of birefringent filters in use now for the study of the solar chromosphere is more than 20 , according to the useful list of solar instruments compiled by R. Coutrez ( $\mathrm{x}$ ) and according to later information received. The Meudon Observatory supervising still the fabrication in Paris (OPL) of the original Lyot filters with an effective band width of $0.75 \AA$ has made considerable efforts in trying to standardize an equipment which could be recommended for automatic solar surveys. As a result of the intimate collaboration with Secasi in Bordeaux a complete Lyot heliograph for automatic recording of the chromosphere in $\mathrm{H} \alpha$ is now available.

Very satisfactory results from the automatic recording with a Lyot filter are reported by the observatory in Uccle. The excellent performance of the $0.5 \AA \mathrm{H} \alpha$ monochromator constructed by A. B. Severny is demonstrated by some photographs of flares which have been published (2). Important improvements of the birefringent filter have been made also by J. W. Evans at the Sacramento Peak Station. For further information as to new instruments in this field reference is made to the report of Sub-commission II $a$.

The Stockholm Observatory reports successful preliminary observations of chromospheric faculae with a birefringent filter for the $\mathrm{CaII} H$ line with an effective band of $2 \cdot 0 \AA$. The filter has been made by Halle Nachfl. in Berlin.

Shortly before his death Dr Lyot made another important invention, the photoelectric coronameter, which is now being perfected at the Meudon Observatory. At the last General Assembly a sum of 2000 dollars was granted for this construction. The new instrument is expected to be ready before the next General Assembly.

M. d'Azambuja reports that his important device, the spectroheliograph with two 
cameras, is being used now with very great success. It enables him to obtain simultaneously spectroheliograms in two different spectral lines or in different parts of one and the same line. The dispersion is $0.6 \AA / \mathrm{mm}$ in the violet. The instrument is particularly valuable for the study of rapidly changing structures.

An important link in the world-wide net of coronagraphic stations was obtained with the installation of a coronograph on Mt Norikura in Japan. Regular observations of the green coronal line are made here since I95I and are published in the Quarterly Bulletin. A new type of Thallium-lamp photometer has been designed for this work (3).

The Sacramento Peak station in New Mexico has new powerful instruments in operation and is publishing since I953 line intensities for $\lambda \lambda 5303,6374$ and $6702 \AA$ in the Quarterly Bulletin.

At the Swedish solar station on Capri a visual photometer with some new features has been tried for the measurements of line intensities in the corona and prominences (Vistas in Astronomy). As in Lyot's photometer in use on Pic du Midi an artificial line produced by solar radiation is superimposed on the continuous spectrum and compared with the emission line. When measuring the centre of the sun a secondary slit of the same width is used in order to transmit the proper wave-length of the continuous spectrum and is compared with the artificial line.

A similar photometer constructed by us for producing artificial bright areas on the solar disk is being used by Mrs K. Lodén in a photometric study of faculae. Preparations are being made to use the same arrangement for measuring intensities and areas of flares and chromospheric faculae.

Mrs Brück reports improvements in the instrumental equipment of the Dunsink Observatory which will give new possibilities for the study of flares and prominences. For the spectrographic work a 7-metre concave grating spectrograph is being used. As to new interesting instrumental arrangements at the McMath-Hulbert Observatory, reference is made to The Sun (4). A new vacuum spectrograph has been added to the equipment.

\section{INTERNATIONAL CO-OPERATION FOR A CONTINUOUS OBSERVATION OF THE Chromosphere and the Prominences}

M. d'Azambuja gives an extensive report in connexion with the preparation of new series of Cartes synoptiques de la chromosphère solaire. Fasc. IX, published in January I952, completed Vol. I, comprising the years I93I to I944. Together with the earlier series (rgr9-30), published in Vol. 6 of Annales de l'Observatoire de Meudon, 346 synoptic maps have been published with not less than 13,752 filaments.

Before starting the edition of Vol. 2 it has been found appropriate to make certain changes in the original conventions first adopted. Already with the special publication by M. and Mme d'Azambuja (5) in 1948 the principal improvements for the new series are indicated. In this work the material for the period I9I9-30 was discussed in detail with special regard to the evolution of filaments. In the same volume tables were presented giving 'centres of activity' and 'characteristic figures' based on the total length of the visible filaments.

A similar supplement has been completed now for Vol. I by Mme d'Azambuja in the case of the centres of activity and by $M$. Dizer in the case of the prominence activity. M. Dizer has completed also for the years I93I-44 a table giving the 'sudden disappearances of filaments'.

The first part of Vol. 2 will appear in I955. It contains solar data for the years I945, I946, and 1947. In the new synoptic maps the centres of activity are indicated. The heights of such filaments which have been observed at the limb are also presented. In this way a more complete information about their real shape may be obtained.

The Meudon Observatory has carried on as before the important work in connexion with the centralization of solar flare observations. On an average, $\mathrm{r}_{4}$ different observatories have been participating. The lists of flares and the maps giving effective observational 
hours have been published in the Quarterly Bulletin on Solar Activity edited by the Zürich Observatory. The numbers of flares were I72 in $1952,9 \mathrm{I}$ in 1953 and 8 only during the first half of I954.

Several solar observatories publish bulletins of a more or less preliminary character and based mainly on their own observations. These bulletins have a great value in addition to the Cartes synoptiques and the Quarterly Bulletin as they are generally printed with short delay. Among these may be mentioned particularly:

Carte-Planisphère de la chromosphère et des taches, published regularly in L'Astronomie by the Meudon Observatory.

Solar Activity Summary, published weekly as well as quarterly by the High Altitude Observatory, Boulder, Colorado. This summary gives a short description of every activity region and contains maps with corona isophotes and flare data according to observations made at the High Altitude Observatory and its station on Climax and the Sacramento Peak station.

Sonnenzirkular, published by the Fraunhofer Institut, Freiburg i. Br. This bulletin gives corona isophotes according to observations made on Wendelstein and Kanzelhöhe. It gives estimated intensities of faculae in integrated light, in $\mathrm{H} \alpha$ and in $\mathrm{K}$, together with data of flares and spots. Kiepenheuer reports that he plans to extend this bulletin with daily maps of the sun giving filaments, chromospheric faculae, prominences, spots and the shape of the corona at the limb. The maps will be distributed twice a month.

Ursigrammes, transmitted by Bureau Ionosphèrique Français (radio station of Pontoise) in collaboration with the Meudon Observatory and with Pic du Midi. Since I949 these transmissions give daily informations about the solar activity. Similar broadcasts of coded messages are made from Kodaikanal (All-India Meteorological Broadcasting Centre, New-Delhi), Darmstadt (Fernmeldetechnisches Zentralamt), and from Tokyo (Astronomical Observatory).

Among important publications of quarterly or yearly character may be mentioned the Bulletin of Solar Phenomena, published by Tokyo Astronomical Observatory, giving character figures of chromospheric faculae, prominences, filaments, etc., and the Osservazioni e Memorie dell'Osservatorio Astrofisico di Arcetri, with regular intormations about areas, heights and intensities of prominences, height of the chromosphere, etc. Annali di Geofisica, Roma, where characteristic numbers of hydrogen and calcium faculae are published according to observations made in Arcetri. As to daily records of prominences at the limb, reference is made to Boletin Astronomico del Osservatorio de Madrid and to the new bulletin published by the observatory in Uccle, Observations de la Photosphère et de la Chromosphere.

Several members of the commission have raised the question as to the best way of intensifying the international solar patrol during the new sunspot cycle. The plans for the geophysical year $1957-58$ give further reason for considering this question. $K$. O. Kiepenheuer points out that we need a better measure of the solar activity than that given by the relative sunspot number, and he recommends that such a measure preferably should be based on the intensity of chromospheric faculae and on the intensity of the solar corona as inferred from line photometry and radioastronomical observations in the Io $\mathrm{cm}$. wave-length. In addition to the apparent activity index, an index characterizing the whole solar surface would be of astronomical interest. Reference is made here to the corresponding recommendation in the report of the sub-commission.

The importance of using in a more exact way the information yielded by the intensity and size of the chromospheric faculae has certainly occurred to many flare observers. A modest attempt along a similar line has been made at the Stockholm Observatory in the classification of faculae in such of high intensity and such of moderate intensity (6). A good solution to this problem no doubt requires special photometric devices. Kiepenheuer and Bruzek are developing for this purpose a simple photometer. The importance of improving the photometric procedures used in investigations of chromospheric phenomena is very much emphasized by $G$. Abetti and $O$. Mohler.

Abetti draws attention also to the fact that 'though we have certainly good methods 
of observing prominences, there is at present no satisfactory way to convey information on their occurrence'. One way would perhaps be to publish daily solar maps giving not only chromospheric phenomena and the shape of the corona but also the prominences at the limb, with special indications of surges, other suddenly appearing objects of high intensity and sudden disappearances.

Kiepenheuer's announcement of a possible distribution from Fraunhofer Institut of daily solar maps is of great interest in this connexion. According to present plans these maps would be based on observations made at several institutions. Abetti makes a similar proposal, but recommends that the central station should collect daily Ursigrams of the various nations and other information by wire or mail and draw every day synoptical maps. In referring to the important Ursigram service already existing, it seems to the chairman that a possible distribution of daily solar maps by telephoto or similar telesystems, as used by many meteorological offices in their daily routine, should be considered in a future world-wide solar information service. Such maps could perhaps be transmitted with a delay of only one day, still giving the central bureau the possibility of collecting informations in addition to their own observations as indicated by Abetti. The participating observatories should be asked to send in corrections and complementary data for a final publication of the definitive maps, preferably as a supplement to the Quarterly Bulletin and the Cartes synoptiques. It seems desirable that the bureau were identical with or had intimate connexions with the central bureau for the cinematography of chromospheric and prominence phenomena. (Compare suggestions made by the subcommission.) If an extension of the present solar information service could be made according to this or a similar scheme, it would probably meet the wishes expressed also by the Commission for the Geophysical Year. But a full realization of such a project would mean the solution first of financial questions too.

A very important work has been carried out since last General Assembly by the working group on Flare Classification. Dr Ellison as chairman of this committee gives the report of their work with recommendations for the future standardization of flare observations (pp. I46-I54 below).

\section{InTERnATIONAL CO-OPERATION FOR Regular OBSERvations of The CoRONa}

The observatories participating at present in the international co-operation for regular corona observations and publishing their results in the Quarterly Bulletin edited by the Zürich Observatory are Arosa, Pic du Midi, Climax, Kanzelhöhe, Mt Norikura, Sacramento Peak and Wendelstein.

As observations of corona line intensities without eclipse are among the most difficult astronomical observations made, it is natural that systematic differences appear between the series of the different stations and that these systematic differences vary from one period of observations to another making the formations of satisfactory mean values almost impossible. In his report $M$. Waldmeier lays stress upon the urgent need of improvements in the methods of standardization, so that average values can be given in the Quarterly Bulletin instead of the individual observations.

This important question was discussed by Commission Mixte pour l'Etude des Relations entre les Phénomènes Solaires et Terrestres at their meeting in Rome in 1952, and a working group was formed in order to find improvements in the photometric procedure. For this purpose J. Rösch and M. Hugon have undertaken, at the observatory on Pic du Midi, a comparison of photographic and visual observations made simultaneously, but because of the low solar activity during recent years this valuable work has taken more time than expected. Of great interest in this connexion is a comparison made recently by $\mathrm{Y}$. Nakagomi and $\mathrm{K}$. Nishi (7) of the photometric scales used at Pic du Midi and Mt Norikura. After reductions for a small error of the standard filter first used on Mt Norikura, the agreement between the two systems is found to be very satisfactory indeed. Another recent study of the relationships between the photometric scales of different coronagraphic stations has been made by S. Nagasawa and H. Morishita (8). W. Kraul has made recently a calibration of the scale used on Wendelstein (9). 
Great expectations are expressed by several members (Abetti, M. d'Azambuja, Kiepenheuer) in connexion with the realization of the photoelectric coronameter for which the Union has granted a sum of 2000 dollars (compare p. 136 above). It is generally felt that this instrument should prove very convenient when deriving contours of the corona in the green line. With reference to the desirable intensification of the general solar patrol during the geophysical year, M. d'Azambuja expects that several instruments of this type will be in operation already in 1957 .

Though the coronameter probably will prove superior to the coronagraph for the photometric survey of the 5303 line, the spectrographic observations with coronagraph will certainly still be of a very great value when controlling these recordings by line photometry, or monochromatic pictures and for the study of other emission lines. A release of some coronagraphs for special investigations would in fact be desirable. Attention may be drawn here to the patrol of the yellow emission line $\lambda 5694$ often associated with flares (xo). Another problem which attracts attention is the recording of white-light streamers of the corona by studying more regularly its polarization. An intimate collaboration with radio astronomy might prove desirable in such a work. The recent announcement by $A$. K. Das and $B$. N. Bhargava (xx) that radio bursts originate not only from flare activity regions (as is well known from the important work by Miss Dodson and others) but sometimes even from $M$ regions, may indicate sudden appearances of white-light streamers. From a careful photometric discussion of plates secured at the eclipse in I9I4 J. Ramberg (12) has found a rapid change in the isophotes which may be of this kind.

\section{PROGREsS OF RESEARCH}

The scientific research carried out since last General Assembly in the field covered by Commission II is considerable. It is not possible therefore to give here a quotation of every paper with complete references. Only a summary is presented with general remarks as to particularly interesting investigations of a general interest.

\section{Chromosphere: general problems}

The temperature of the chromosphere is still a question which attracts much attention. With reference to the presentation by van de Hulst (The Sun) of different arguments for a high-temperature scale and a low-temperature scale it can be mentioned that I. Kawaguchi (Kyoto University) finds a kinetic temperature of $10,000^{\circ} \mathrm{K}$. from a study of hydrogen emission-line contours. S. Miyamoto (Kyoto University, unpublished) has made an interpretation of the observed intensity and width of the solar Lyman $\alpha$ radiation suggesting a chromospheric temperature of $6,000^{\circ} \mathrm{K}$. V. A. Krat (13) has studied the scattering process connected with the formation of hydrogen, helium and calcium II lines, obtaining a temperature which does not exceed $6000^{\circ} \mathrm{K}$ for heights less than $45^{\circ} 00 \mathrm{~km}$. Unsöld (14) finds that the temperature corresponding to the excitation of low energy states $\left(\mathrm{H} \alpha\right.$, etc.) is of the order of $3800^{\circ} \mathrm{K}$.

The study of the fine structure of the chromosphere at the limb is in progress at Climax, Sacramento Peak and Poulkovo. V. A. Krat and I. A. Prokofieva (15) conclude that the chromosphere consists of a number of small prominences. According to their results the gradient of atomic numbers in the chromosphere is connected with the number of fine structure prominences along the line of sight and with the height of these prominences, but not with the density distribution. T. V. Krat finds that the intensity gradient is two times smaller (scale height larger) above the faculae than above the undisturbed part of the solar limb.

In Meudon M. Dizer has studied chromospheric spicules on films secured by Dr Lyot, whereas Miss S. Lippincott has studied on his chromospheric films the filamentary fine structure between sunspots. Reference is made to the report of Sub-commission II $a$.

Astrophysical investigations of chromospheric faculae have been made at several institutions. G. Righini has measured in Arcetri the width of $\mathrm{H} \alpha$ in such objects. 
E. R. Murstel (x6) has shown that electronic inpact is probably responsible for the emission of the $\mathrm{H}$ and $\mathrm{K}$ lines in chromospheric faculae. According to his results the large width of $\mathrm{H}_{2}$ and $\mathrm{K}_{2}$ cannot be explained as non-coherent scattering, but is connected with selfabsorption. The presence of $\mathrm{H}_{3}$ and $\mathrm{K}_{3}$ is explained by a decrease of $\mathrm{T}_{\mathrm{kin}}$ upwards.

In the case of the central part of the $\mathrm{H} \alpha$ line the process of recombination is responsible for the emission. This would explain the difference between spectroheliograms obtained in $\mathrm{H} \alpha$ and in the $\mathrm{H}$ and $\mathrm{K}$ lines. Only in the bright $\mathrm{H} \alpha$-chromospheric-faculae the role of electronic impact may be important.

Contributions to the theory of spectroheliograms has been given also by M. Reichel at the Kiel Observatory. Following the interpretation given before by Unsöld, Reichel finds that the temperature is higher in the faculae than in undisturbed regions for optical depths $<0.8$ whereas the opposite effect is present in optical depths $>0.8$. With this model many characteristics of the chromospheric faculae can be explained too.

\section{Solar flares, surges and activation of filaments (prominences)}

The application in the study of flares of strictly photometric principles has been found a very fruitful programme. M. A. Ellison has continued his extensive work in this field at Edinburgh, Miss Dodson has derived important results from similar work at the McMath-Hulbert Observatory and Miss Ballario has studied accurate light curves of flares in $\mathrm{H} \alpha$ and $\mathrm{K}_{23}$ observed at Meudon and Arcetri.

A recent result found by Miss Dodson (17) which is important to remember in future flare patrol observations as well as in theoretical studies is the correlation between line width in $\mathrm{H} \alpha$ and the distance of the flare from the central meridian. For flares of importance 2 and 3 the average width of $\mathrm{H} \alpha$ increases from $3.6 \AA$ near the central meridian to $6 \cdot 0 \AA$ near the limb.

Another very interesting field of investigation is the simultaneous application of optical and radioastronomical observations in the study of flares. Miss Dodson(18) reports that the basic forms of the flare-associated events on the wave-lengths $\mathrm{I} \cdot 5 \mathrm{~m}$. (Cornell) and $10.7 \mathrm{~cm}$. (Ottawa) seem to be fundamentally similar for the flares studied (I94 and 386 respectively). The fully developed flare-associated radio event exhibits two parts, but either of the two parts may occur separately. At $1.5 \mathrm{~m}$. the first or 'early' part of the double pattern is a sudden major burst which starts with the beginning of the flare and is usually over by the time $\mathrm{H} \alpha$ maximum has been attained. The second or 'late' part starts more gradually and attains its maximum as the flare is fading over. When fully developed, this second part appears to be identical with the feature known as 'noise storm'.

At $10.7 \mathrm{~cm}$. the first or 'early' part is a sudden single burst which may be either simple or complex in structure. This burst starts with the start of the $\mathrm{H} \alpha$ flare and is usually over by the time $\mathrm{H} \alpha$ maximum has been attained. The 'second part' is a gradual diminution or rise and fall of flux. At ro.7 cm. the 'second part' seems to start simultaneously with the flare, contrary to its late aspect at $\mathrm{r} \cdot 5 \mathrm{~m}$.

A higher percentage of flares $(78 \%)$ was apparently associated with some form of distinctive event at $\mathrm{r} \cdot 5 \mathrm{~m}$. than was the case $(40 \%)$ at $10.7 \mathrm{~cm}$. At $\mathrm{r} \cdot 5 \mathrm{~m}$. the percentage was practically independent of flare importance, but at $10.7 \mathrm{~cm}$. the percentage increased from $19 \%$ for subflares to $87 \%$ for flares of importance 2 and 3 . There is strong evidence that all outstanding disturbances at $10.7 \mathrm{~cm}$. are associated in time with the occurrence of flares.

A. B. Severny and E. F. Shaposhnikova $(x)$ have carried out an investigation of more than Ioo flares with the aid of a narrow band $\mathrm{H} \alpha$ filter. It was observed that on the average one flare appears every seven hours of the spot's lifetime. More than $30 \%$ of flares show motions that are quite similar to the motions of 'electromagnetic' prominences with velocities up to $300 \mathrm{~km}$. $/ \mathrm{sec}$. Almost all flares show a simultaneous growth of intensity and area. As a possible source of energy a mechanism of Joules' heating is discussed. 
In another paper (20), relating to the limb flare of Aug. I6, I952, Severny discusses nuclear processes as source of energy-a possibility hinted at in I 946 by Saha. Severny reports that N. M. Barabashev and J. M. Gordon consider the possible existence of 'unvisible' flares. Gordon advances the hypothesis that relativistic electrons appearing in magnetic fields of spots may give the high energy of flares.

A critical discussion of the discharge theory of flares as developed by Giovanelli and others has recently been published by T. G. Cowling (2r).

Extensive observations and theoretical studies of flares have been made at the astronomical institute in Ondrejov and Prahy. Z. Svestka has discussed line profiles and determined temperatures and densities by making different assumptions as to the broadening mechanism responsible for the width of the $\mathrm{H} \alpha$ line. He has made also a statistical investigation of the appearance of flares and found that revived centres of activity show a remarkably high frequency of flares. Some striking examples of this interesting effect have been noted also at the Swedish Solar Station on Capri. According to Svestka the relatively small flare activity during last sunspot cycle compared with the high number of spots may be due to the small number of revived centres.

F. Link and J. Kleczek have continued in Ondrejov the study of the asymmetrical distribution of flares in heliographic longitude. Different statistical investigations have been made by Kleczek and by C. J. Macris in Athens.

The dark filaments (surges) accompanying solar flares have been studied recently from an extensive material by $\mathrm{T}$. Tsumita and $\mathrm{K}$. Mizugaki of the Tokyo Observatory. From measurements of sight-line velocities they conclude that the negative velocities first increase (numerically) and then, after attaining a maximum, diminish to zero. Already at their first appearance the positive velocities exceed the negative ones. After about 20 minutes a maximum velocity is attained and the values decrease slowly. After an hour or so only the positive velocities remain. The results seem to be in substantial agreement with earlier results found by $\mathrm{H}$. W. Newton.

A. Bruzek has secured from many years' work on Kanzelhöhe an interesting material showing the influence of flares on existing filaments. According to Bruxek there are two kinds of influences. One displays itself as an activation of the filament by some kind of perturbation travelling with a speed of about Ioo $\mathrm{km}$. $/ \mathrm{sec}$. This effect is probably related to a phenomenon discussed by R. S. Richardson in 1935 and I95I from material secured at the Mount Wilson Observatory, that flares have a tendency sometimes to appear more or less simultaneously in different spot groups. A few examples suggesting travelling disturbances of similar kind have been described recently in reports from the Swedish Solar Station on Capri.

Another effect of long distance disturbances is displayed by the sudden disappearances of filaments well known from the pioneer work of M. d'Azambuja. According to Bruzek the disturbance is here connected with the formation of a new spot group and is propagated with much lower speed, only about I $\mathrm{km}$. $/ \mathrm{sec}$.

\section{Prominences in general}

The description of the physical conditions of prominences by a certain temperature often leads to apparent divergences between the results of different scientists. A very clear statement in papers published on this subject of the kind of temperature obtained and the kind of object examined is now more important than ever. Formation of mean values should also be made with great care. This is exemplified by an interesting recent result found by D. E. Billings (Boulder) that line spectrum analysis of prominences may give sometimes as high kinetic temperatures as hundreds of thousands of degrees. For very transparent objects, more or less of the same character as the corona itself, such a result is understandable. For more opaque objects such a high temperature would generally mean an unreasonable brightness. Referring to the fact that most prominences appear as dark filaments on $\mathrm{H} \alpha$ spectroheliograms, Unsöld (22) emphasizes with stress that the electron temperature must be of the order of $3800^{\circ} \mathrm{K}$. Still lower temperatures 
have been given by $\mathrm{K}$. Wurm for the darkest filaments and are also indicated by lowintensity filaments visible against bright limb prominences according to descriptions made by Öhman (23) in several publications. It seems to the chairman that apparently conflicting results in this connexion may be found consistent by considering the prominences as composed of a great many narrow filamentary elements of low temperature and high density embedded in a cloud of very high temperature and low density. In fact a filamentary fine structure is often indicated from observations during favourable observing conditions as have been found by many observers. Reference is made to similar questions discussed on p. I40 above. It should be quoted here, however, that L. Woltjer (24) arrives at the conclusion that the spicules are columns of hot gas in a cooler chromosphere.

Severny reports extensive work on prominences carried out at the Simeis and Partizanskoe observatories (compare the report of the sub-commission). The prominences are classified as eruptive, electromagnetic and chaotic according to the type of predominant motion. The motions of the electromagnetic ones are interpreted according to the principles developed by $\mathrm{H}$. Alfvén (25). Another, though related, system of classification was presented at the Volta Conference of 1952 by D. H. Menzel and J. W. Evans.

At the Kodaikanal Observatory Ananthakrishnan has made a statistical investigation of the distribution of prominences in latitude during four sunspot cycles by using observations made in Kodaikanal in the period I905-50. Together with A. K. Das he has studied an east-west asymmetry in the appearance of $\mathrm{H} \alpha$ filaments.

Das (26) reports an interesting observation of radio noise burst in connexion with the development of an eruptive limb prominence not connected with any solar flare. (Compare Dodson and Donselman (27).) This observation may be compared with eclipse observations in 1952 made by $\mathrm{O}$. Rydbeck (28), indicating radio emission from an extended filament.

Considerable work has been done in determining line contours of prominences (M. A. Ellison, G. S. Ivanov-Kholodny, V. L. Khochlova and J. J. Nazarova, G. Righini and others). Reference is made to the report of Commission I2. A study of intensity-time relationships for $\mathrm{H} \alpha$ and $\mathrm{D}_{3}$ in selected prominences is being made by $F$. Lindgren (Stockholm Observatory).

\section{The corona}

M. Trellis has carried out a determination of the rotation of the lower parts of the corona by using the accurate line intensity measurements made on Pic du Midi. He has been able to establish a relationship between angular rotation and latitude similar to that found by M. and Mme d'Azambuja for the filaments. From a study of the distribution of corona jets Trellis concludes that the new cycle started already in I95 I at a latitude of about $70^{\circ}$ for $\lambda 5303$. With a new birefringent filter calculated by $\mathrm{A}$. Dollfus which can be used for $\lambda 5303$ as well as for $\lambda 6374$ and which has a passband of $3 \AA$, J. Rösch and his collaborators have obtained good photographs of the strong corona jet visible during October 1954 .

The yellow corona line $\lambda 5694$, which according to earlier work made by B. Lyot, W. O. Roberts, M. Waldmeier and others seems to be a characteristic feature of regions of high activity, has been the subject of new studies at the High Altitude Observatory in Boulder. Further evidence has been obtained that this line is clearly associated with solar flares (29) and with active region prominences (30). According to work carried out at the same institution (31) the atomic weight of the element responsible for this line is about 40 , which agrees well with the original identification made by $\mathrm{B}$. Edlen that the line is due to CaXv.

A. Dollfus also reports some observations of the yellow line associated with eruptive prominences (Volta Conference, I952). He has found as well that the green line $\lambda 5303$ shows sometimes a remarkably high intensity around eruptive prominences. The President wants to draw attention here to a classical paper by Trouvelot (32) describing spectroscopic observation (without eclipse) of the green line in some extremely bright prominences. It is perhaps not impossible that Trouvelot really saw the line! Trouvelot's paper contains a description of several objects giving sudden geomagnetic disturbances. 
Of interest not only to Commission $x_{3}$ but also to Commission II are results of the French eclipse expeditions to Khartoum, published recently by Dollfus. He presents a list of new corona lines present on the plates secured by Lyot (33), and he has completed now a discussion of the monochromatic pictures obtained in the red and green corona lines. Other results from solar eclipse observations which may be mentioned here are those obtained by $\mathrm{H}$. von Klüber (34), by J. Evans (35) of the American expedition I952, and by J. Ramberg and H. Kristenson, covering eclipses in Sweden in I9I4 and I945 (36). A special attention may be drawn also to results found in 1952 by A. Colacevich (37) that the $\mathrm{H}$ and $\mathrm{K}$ emission lines can be traced in the outer corona. In his report Severny announces that M. N. Gnevyshev (38) has observed well-pronounced emission of $\mathrm{H}$ and $\mathrm{K}$ lines of $\mathrm{CaII}$ in the spectrum of the inner corona above regions occupied by faculae. Attempts by Y. Ohman to observe the corona at the eclipse of 30 June I954 with a narrow Carr $\mathrm{H}$ line monochromator failed because of cloudy sky. If $\mathrm{H}$ and $\mathrm{K}$ emission is present, this emission is likely to be due to fragments of prominences and not to the hot corona gas itself.

The large undisturbed regions of the corona called $M$ regions and identified with the corpuscular source of certain geomagnetic disturbances, have been studied by W. $\mathrm{O}$. Roberts, D. E. Billings, J. C. Pecker and others (39) in Boulder, and by A. Bruzek (40) in Freiburg. Roberts and his collaborators associate the corpuscular source with long white-light corona streamers (quoted already on p. 140 ). Bruzek finds that the $M$ regions are characterized by abnormally small intensities of the green and red corona lines.

Bruzek (4x) has found that there is a very pronounced correlation between the intensity and extension of corona emission regions as revealed by the green line and the intensity and extension of chromospheric faculae. The Ir-year variation of the total intensity of the corona as revealed by the $\lambda 5303$ and $\lambda 6374$ lines has been studied by M. Waldmeier.

Measurements of the decrease in intensity of the green corona line with increasing distance from the limb has been made in $195^{2}$ by M. Notuki and I. Shimizu (42) of the Tokyo Observatory. The logarithmic gradient is in substantial agreement with eclipse observations for this line made in I940 by C. W. Allen (43).

\section{SUgGestions MADE BY MEMbers of The UNion}

I. M. d'Azambuja and Coutrez recommend an intensification in the world-wide solar patrol in order to meet the request of complete solar data, as advanced for instance by the Commission for the Geophysical Year.

2. Abetti, Coutrez, Kiepenheuer, Mohler, and Waldmeier recommend improvements in photometric measurements, notably in the co-operative determination of corona line intensities. The use of several Lyot coronameters probably gives the best solution for the future.

3. Kiepenheuer suggests a co-operation in photometric photometry of chromospheric faculae in order to find a new adequate index of solar activity.

4. Unsöld recommends an intensified study and a more complete recording of surges. Observations of these objects are of particular interest in connexion with radio burst phenomena.

5. Öhman recommends a continuous recording not only of solar disk phenomena, but of limb prominences as well, by making every tenth exposure with the automatic narrow pass band filters longer than the ordinary exposures.

6. Ohman suggests that a list should be published in Quarterly Bulletin of sudden appearances of high-intensity limb prominences. Such objects often produce ionospheric and similar disturbances, and associated flares may escape direct observation.

7. Ohman draws attention to the need of more regular observations of white-light streamers of the corona and thinks that those coronagraphs eventually released from the present routine programme, with the construction of Lyot coronameters, might find valuable use in such a work.

8. Coutrez and Kiepenheuer draw attention to the difficulty in finding commercial $\mathrm{H} \alpha$ films for the automatic recordings. 
9. C. de Jager asks the commission to recommend a proper nomenclature for the fine structure on spectroheliograms.

Io. Abetti, Kiepenheuer, Roberts and Öhman raise the question of how to find satisfactory ways of transmitting daily solar information maps in addition to the present Ursigram service.

II. Recommendations made in the special report of the working group on Flare Classification (see below).

\section{ReCommended Subventions}

I. Of the grant to Meudon M. d'Azambuja says:

L'Observatoire de Meudon demande que la subvention annuelle de 2.700 francs-or, accordée depuis I925 par l'U.A.I. à l'Observatoire de Meudon pour la publication des Cartes synoptiques de la chromosphère solaire, soit renouvelée pour la période qui s'écoulera jusqu'à la prochaine Assemblée générale.

2. The commission strongly recommends the renewal of the subvention to the Meudon Observatory as requested by M. d'Azambuja.

\section{REFERENCES}

Y. ÖHMAN

President of the Commission

(I) R. Coutrez, in The Sun, ed. Kuiper (Chicago Univ. Press, 1953).

(2) Astr. J. U.S.S.R. 31, no. 2, 123 , 1954.

(3) Ann. Tokyo Astr. Obs. 2nd ser., 3, 182, 1953.

(4) The Sun, ed. Kuiper, p. 605, 1948.

(5) L. and M. L. d'Azambuja, Ann. Meudon, 6, fasc. 7, 1948.

(6) Tellus, in the Press.

(7) Y. Nakagomi and K. Nishi, Tokyo Astr. Obs. Report, 10, 264, 1953.

(8) S. Nagasawa and H. Morishita, Tokyo Astr. Obs. Report, 1o, 26I, 1953.

(9) W. Kraul, $Z$. AP. 33, I74, 1953.

(10) F. P. Dolder, W. O. Roberts, D. E. Billings, Ap. J. 119, 120, 1954.

(I I) A. K. Das and B. N. Bhargava, Nature, 172, 855, 1953.

(12) J. Ramberg, Stockholm Obs. Ann. Vol. r6, 3, I95I.

(13) V. A. Krat, C.R. Acad. Sci. U.R.S.S. 1954.

(I4) A. Unsöld, Physik der Sternatmosphären (new ed., in the Press).

(15) V. A. Krat and I. A. Prokofieva, Pulkovo Bull. no. 152.

(16) E. R. Mustel, Publ. Crim. Astrophys. Obs. 1952 and 1954.

(17) H. W. Dodson, $A p . J$. 120, 83, 1954.

(18) H. W. Dodson, $A p . J$. 118, I69, 1953 and rr9, 54I, I954.

(19) A. B. Severny and E. F. Shaposhnikova, Publ. Crim. Astrophys. Obs. 12, 3, r954.

(20) A. B. Severny, Publ. Crim. Astrophys. Obs. Vol. r3, 1954.

(2I) T. G. Cowling, in The Sun, ed. Kuiper, P. $5^{87}$.

(22) A. Unsöld, Physik der Sternatmospharen (new ed., in the Press).

(23) Y. Öhman, Atti del Convegno Volta, I953.

(24) L. Woltjer, B.A.N. no. 454, 12, 165, 1954.

(25) H. Alfvén, Cosmical Electrodynamics, Oxford, r950.

(26) A. K. Das, Nature, 172, 446, 1953.

(27) H. W. Dodson and R. Donselman, $A p . J$. Ir3, 519, I95I.

(28) O. Rydbeck, Atti del Convegno Volta, I953.

(29) $A p . J$. 119, r20, 1954.

(30) $A$ P.J. 120, I12, 1954.

(3I) C.R. 238, I I94, 1954 .

(32) E. L. Trouvelot, Bull. Soc. Astr. France, 7, 19, 1893.

(33) A. Dollfus, C.R. 237, 854, 1953.

(34) H. von Klüber, The Observatory, 72, 207, 1952. 
(35) J. Evans, $A p . J$. 120, 94, I954.

(36) J. Ramberg, Stockh. Obs. Ann. 17, No. 2, 1951; H. Kristenson, ibid. 18, No. 5, 1955.

(37) A. Colacevich, Atti del Convegno Volta, r953.

(38) M. N. Gnevyshev, C.R. Acad. Sci. U.R.S.S. 94, 811 , 1954.

(39) W. O. Roberts et al., Astr. J. 58, 225, 1953 (and in the Press).

(40) A. Bruzek, $Z$. Naturf. 7a, 708, r952.

(4I) A. Bruzek, $Z$. Ap. (in the Press).

(42) M. Notuki and I. Shimizu, Tokyo Observatory Report, ro, 258, 1953.

(43) C. W. Allen, M.N. 106, I36, I946.

\section{APPENDIX}

\section{REPORT OF THE WORKING GROUP ON FLARE CLASSIFICATION}

During the Eighth General Assembly of the International Astronomical Union held at Rome in September I952, it was resolved to appoint a Working Group of Commission II to report on improved methods of flare classification. The members of this committee were: L. d'Azambuja, Marguerite d'Azambuja, Helen Dodson, M. A. Ellison (chairman), H. W. Newton and A. Severny. A great volume of observational material has had to be analysed in the preparation of this report, and I acknowledge with gratitude the generous and indispensable co-operation of these individuals, as well as that of Dr P. A. Wayman of Herstmonceux.

Twenty years have elapsed since it was planned to organize the observation of solar flares on an international basis, under the auspices of the I.A.U.(x). During this period flares have been classified on a scale of importance- $I$ (smallest), 2 (intermediate), 3 (largest); the further category $3+$ (introduced by Newton) is also in general use to denote flares of outstanding importance. In addition, many observers use intermediate steps, such as I + or 2 - to indicate their estimate that the flare exceeds importance I but is less than 2. Although it was recommended in 1935 that estimates should be based upon area alone, the scale of importance has remained very largely a subjective scale: it has always been left to the discretion of individual observers to decide to what extent their estimates of importance should be based upon quantitative measures.

Consequently, the essential problem before the committee has been to review the existing situation and to decide:(a) how many steps in the importance scale are desirable, (b) to what extent, and in what way, the estimates should be based upon quantitative measures, such as area, line-width or central intensity of the $\mathrm{H} x$ emission, and $(c)$ to provide simple guidance in these matters for observers who use different instruments and different methods of observation.

In the early days, flare patrols were carried out visually by means of spectrohelioscopes, -usually of the Hale pattern, and this method is still widely employed. More recently, automatic spectroheliographs and Lyot filters employing cinematography have been introduced. The present multiplicity of instruments increases the difficulties of our problem, since, for example, the measured central intensities of $\mathrm{H} \alpha$ in a flare stand in need of considerable correction, and these corrections can only be applied if the constants of each instrument have been determined. The corrections are a function both of the instrument and of the brightness of the flare.

The quantities amenable to measurement are as follows:

(a) duration of the flare from start to finish;

(b) area of the region, or regions, of enhanced emission in $\mathrm{H} \alpha$;

(c) effective line-width of $\mathrm{H} \alpha$ in angstroms at the brightest point;

(d) the central intensity of $\mathrm{H} \alpha$, expressed as a fraction of the level of the continuous spectrum outside the line, or in terms of the background intensity of the Fraunhofer $\mathrm{H} \alpha$.

Of these, $(d)$ is perhaps the most difficult to determine because of the short duration of maximum intensity and of the uncertainty of the instrumental corrections involved. 